\title{
Radiofrequency ablation for cholangiocarcinoma: Do we need to be more precise?
}

\section{다 (i)}

\author{
Authors \\ Einas Abou Ali ${ }^{1}$, Maximilien Barret ${ }^{1,2}$ \\ Institutions \\ 1 Digestive disease and endoscopic departement, Cochin \\ Hospital, Paris, France \\ 2 Paris Descartes University, Université de Paris, Paris, \\ France \\ Bibliography \\ DOI https://doi.org/10.1055/a-0982-3255 | \\ Endoscopy International Open 2019; 07: E1301-E1302 \\ (c) Georg Thieme Verlag KG Stuttgart · New York \\ elSSN 2196-9736 \\ Corresponding author \\ Maximilien Barret, Endoscopy Unit, Cochin hospital, \\ Gastroenterology Digestive Oncology Department, 27 Rue \\ du Faubourg Saint Jacques, 75014 Paris \\ Fax: +0033158413865 \\ maximilien.barret@aphp.fr
}

Endobiliary radiofrequency ablation (RFA) performed on the biliary tree during endoscopic retrograde cholangiopancreatography (ERCP) is proposed for palliative management of malignant biliary tract obstructions to improve patency of biliary stents [1,2]. However, optimal generator settings in terms of probe length, ablation power and duration are still unclear because of the existence of two different endobiliary RFA catheters and of specific physiological characteristics between the proximal and distal biliary tree. This might account for the high morbidity rates observed after endobiliary RFA, particularly during treatment of hilar obstruction $[3,4]$.

In this issue of Endoscopy International Open, Eui Joo Kim et al. assess the feasibility and safety of endobiliary RFA for treatment of hilar malignant obstruction in both an animal model and a clinical pilot study [5]. They used a 7Fr temperature-controlled catheter (ELRA; STARmed, Goyang, Korea) and a dedicated generator and performed procedures under ERCP guidance. In the preclinical dose-finding study on six healthy minipigs, the authors performed endobiliary RFA in the hilar area using different probe lengths $(11,18$, and $22 \mathrm{~mm}$ ) and settings (7 or $10 \mathrm{~W}$ and 60 to $120 \mathrm{~s}$ ). Only two of the six animals, treated with the smallest probe at the lowest intensity and shortest duration (11 mm, 7W and $60 \mathrm{~s}$ ), did not develop bile duct perforation, although transmural necrosis was found on histology. Based on these findings, the authors performed a clinical pilot trial in 11 patients with malignant hilar obstruction not amenable to surgery. For seven patients, the ablation time was 60 seconds, 120 seconds for the other four, and tandem overlapping endobiliary RFA was attempted in all patients before placement of bilateral metal stents. The technical success rate was $100 \%$, and no early adverse event such as hemobilia or perforation was reported. Only one patient ( $9 \%$ ) had a post-proce- dure mild pancreatitis managed by medical treatment. Onethird of patients $(3 / 11)$ presented with a stent occlusion at 84 , 89 , and 91 days after the procedure, respectively, while the median event-free survival was 91 days $(95 \% \mathrm{Cl}=82-100)$.

In the past 10 years, endoscopic endobiliary radiofrequency ablation has gained momentum and improved technically, and has been assessed for treatment for unresectable malignant biliary hilar or distal obstruction [6], bridge to surgery [7] residual adenoma in the common bile duct after endoscopic papillectomy [8], or even benign refractory biliary strictures [9] Yet, some data remain controversial concerning the benefit of endobiliary RFA in terms of stent patency and survival $[4,6,10]$, mainly due to heterogeneity in types and locations of neoplastic obstruction. The randomized trial conducted by Yang et al. in 65 patients with unresectable extrahepatic cholangiocarcinomas, provided the most specific data on endobiliary RFA, but still included distal and hilar tumors [6]. Of note, the authors demonstrated a statistically significant doubling of stent patency and a $50 \%$ increase in survival in the endobiliary RFA+ stent group vs. the stent-only group. However, most studies, including the one from Eui Joo Kim et al., included pancreatic adenocarcinomas, cholangiocarcinomas, gallbladder cancers, and strictures related to liver metastases. Therefore, a first step would be to better define for which of these diseases and for which location (distal, hilar or intrahepatic) endobiliary RFA is the most effective. It is possible that endobiliary RFA is beneficial for all these patients, but with specific generator settings that would need to be defined in adequate in vivo in animal models [10].

Secondly, pretreatment cartography by cholangioscopy or measurement of biliary wall thickness by intraductal ultrasonography has been proposed to specify the location and length of 
the intraductal lesion. However, it seems unrealistic in clinical practice, as these techniques will significantly increase costs (if not technical difficulty, length and morbidity) of the procedures. Indeed, the question of cost-effectiveness of endobiliary RFA needs to be evaluated. In a palliative setting, is the combination of endobiliary RFA and stenting a relevant medico-economic option compared to repeated ERCP drainage and stenting?

As a conclusion, endobiliary RFA is a promising treatment option for unresectable malignant biliary obstruction. Thanks to studies such as the one published in this issue of Endoscopy International Open, overall safety of the procedure is improving. Further studies will help to determine specific generator settings, clinical benefit, and medico-economic validity of endobiliary radiofrequency ablation for each cause and location of malignant biliary obstructions.

Competing interests

None

\section{References}

[1] Steel AW, Postgate AJ, Khorsandi S et al. Endoscopically applied radiofrequency ablation appears to be safe in the treatment of malignant biliary obstruction. Gastrointest Endosc 2011; 73: 149-153

[2] Sofi AA, Khan MA, Das A et al. Radiofrequency ablation combined with biliary stent placement versus stent placement alone for malig- nant biliary strictures: a systematic review and meta-analysis. Gastrointest Endosc 2018; 87: 944-951.e1

[3] Tal AO, Vermehren J, Friedrich-Rust M et al. Intraductal endoscopic radiofrequency ablation for the treatment of hilar non-resectable malignant bile duct obstruction. World J Gastrointest Endosc 2014; 6: $13-19$

[4] Dolak W, Schreiber F, Schwaighofer $\mathrm{H}$ et al. Endoscopic radiofrequency ablation for malignant biliary obstruction: a nationwide retrospective study of 84 consecutive applications. Surg Endosc 2014; 28: $854-860$

[5] Kim E], Choo JH, Kim Y] et al. Intraductal temperature-controlled radiofrequency ablation in malignant hilar obstruction: A preliminary study in animals and initial human experience. Endosc Int Open 2019; 07: E1293-E1300

[6] Yang J, Wang J, Zhou H et al. Efficacy and safety of endoscopic radiofrequency ablation for unresectable extrahepatic cholangiocarcinoma: a randomized trial. Endoscopy 2018; 50: 751 - 760

[7] Kim EJ, Chung DH, Kim YJ et al. Endobiliary radiofrequency ablation for distal extrahepatic cholangiocarcinoma: A clinicopathological study. PLoS One 2018; 13: e0206694

[8] Camus M, Napoléon B, Vienne A et al. Efficacy and safety of endobiliary radiofrequency ablation for the eradication of residual neoplasia after endoscopic papillectomy: a multicenter prospective study. Gastrointest Endosc 2018; 88: 511 - 518

[9] Akinci D, Unal E, Ciftci TT et al. Endobiliary radiofrequency ablation in the percutaneous management of refractory benign bilioenteric anastomosis strictures. AJR Am J Roentgenol 2019; 212: W83 - W91

[10] Teoh AY, Cheung SY, Chong C et al. Endoscopic biliary radiofrequency ablation for malignant distal common bile duct obstruction doesn not improve survival. A randomized controlled trial. Gastrointest Endosc 2018; 87: AB104-AB105 